\title{
Expansão de classes ordinais em pré-escolares
}

\author{
Lívia Bentes de Souza \\ Mariana Morais Miccione \\ Grauben José Alves de Assis \\ Universidade Federal do Pará-Belém, PA, Brasil
}

\section{Resumo}

Pesquisas desenvolvidas têm documentado a formação de classes ordinais em humanos por meio do ensino de sequências independentes. $\mathrm{O}$ ensino de sequências por sobreposição de pares (e.g. A1 $\rightarrow \mathrm{A} 2$, $\mathrm{A} 2 \rightarrow \mathrm{A} 3, \mathrm{~A} 3 \rightarrow \mathrm{A} 4, \mathrm{~A} 4 \rightarrow \mathrm{A} 5)$ pode ser a base para a formação de relações ordinais intra e entre sequências de forma inequívoca. O objetivo do presente estudo foi replicar o procedimento de ensino por sobreposição com trios de estímulos, a partir da expansão das classes ordinais em três préescolares. Foram utilizados dois conjuntos de estímulos: um com numerais cardinais de 1 a 9 e outro com formas não representacionais. Um software foi utilizado na apresentação de estímulos e registro das respostas. Um notebook com tela de 15' apresentava vídeos animados contingentes a cada acerto. Testes de transitividade (ex. 3-5-7 ou 5-7-9) foram apresentados após o ensino por sobreposição com sequências de três estímulos (ex. 1-2-3, 3-4-5, 5-6-7, 7-8-9). O segundo conjunto de estímulos foi apresentado com a mesma sequência de treino e testes. Em seguida, o teste de conectividade foi aplicado. Resultados demonstraram que os três participantes responderam com $100 \%$ de acerto na maioria dos testes. Esses resultados também sugerem uma linha de base corretamente programada.

Palavras-chave: Relações ordinais, Sobreposição de estímulos, Numerosidade, Pré-escolares.

\section{Expansion of ordinal classes in preschoolers}

\begin{abstract}
Studies conducted have documented the formation of ordinal classes in humans through the teaching of independent sequences. Teaching sequences for overlapping pairs (e.g. A1 $\rightarrow \mathrm{A} 2, \mathrm{~A} 2 \rightarrow \mathrm{A} 3$, $\mathrm{A} 3 \rightarrow \mathrm{A} 4, \mathrm{~A} 4 \rightarrow \mathrm{A} 5$ ) may be the basis for the formation of ordinal relations within and between sequences unequivocally. The objective of this study was to replicate the teaching procedure by overlapping trios of stimuli, from the expansion of ordinal classes in three preschoolers. We used two sets of stimuli: one with cardinal numbers 1 to 9 and another with non-representational forms in these quantities. Software was used in the presentation of stimuli and recording responses. A notebook with 15 ' screen presented animated videos contingent to each hit. Tests of transitivity (eg, 3-5-7 or 5-7-9) were presented after teaching overlap with sequences of three stimuli (eg 1-2-3, 3-4-5, 5-6-7, 7-8-9). The second set of stimuli was presented with the same sequence of training and testing. Then the connectivity test was applied. Results showed that three participants responded with $100 \%$ accuracy in most tests. These results also suggest a baseline programmed correctly.
\end{abstract}

Keywords: Ordinal relations, Overlapping stimuli, Numerosity, Preschoolers.

Endereço para correspondência: Rua Jerônimo Pimentel, 426-1801. Bairro do Umarizal, Belém/PA. CEP: 66055-000. E-mail: ggrauben@gmail.com. Telefone: +55 9132293090 (residencial) / +55 9132018488 (comercial).

Sobre os autores:

Lívia Bentes de Souza - Bolsista do PIBIC-CNPq.

Mariana Morais Miccione - Doutoranda do Programa de Pós-Graduação em Teoria e Pesquisa do Comportamento, Universidade Federal do Pará.

Grauben José Alves de Assis - Doutor em Psicologia pela Universidade de São Paulo. Docente do Programa de Pós-Graduação em Teoria e Pesquisa do Comportamento, Universidade Federal do Pará. Bolsista de Produtividade em Pesquisa do CNPq.

Artigo aceito durante a gestão do editor Gerson Tomanari. 


\section{La expansión de las clases ordinales en niños en edad preescolar}

\section{Resumen}

Estudios realizados han documentado la formación de las clases ordinales en los seres humanos a través de la enseñanza de las secuencias independientes. La enseñanza de secuencias didácticas de pares de superposición (por ejemplo A1 $\rightarrow$ A2, A2 $\rightarrow$ A3, A3 $\rightarrow$ A4, A4 $\rightarrow$ A5) puede ser la base para la formación de las relaciones de orden dentro y entre las secuencias de manera inequívoca. El objetivo de este estudio fue replicar el procedimiento de enseñanza por la superposición con tríos de estímulos, a partir de la expansión de las clases ordinales en tres niños en edad preescolar. Se utilizaron dos series de estímulos: uno con los números cardinales 1 al 9 y otras formas con las organizaciones norepresentacional. Se utilizó un software para la presentación de los estímulos y grabación de las respuestas. Un computador portátil con pantalla de $15^{\prime}$ presentaba videos animados contingente a cada acierto. Las pruebas de transitividad (por ejemplo, 3-5-7 o 5-7-9) se presentaron después de enseñar la superposición con las secuencias de tres estímulos (por ejemplo, 1-2-3, 3-4-5, 5-6-7, 7-8-9). El segundo conjunto de estímulos se hizo con la misma secuencia de entrenamiento y pruebas. Luego se aplicó la prueba de conectividad. Los resultados mostraron que los tres participantes respondieron con un $100 \%$ de precisión en la mayoría de las pruebas. Estos resultados también sugieren una línea de base correctamente programado.

Palabras clave: Las relaciones ordinales, Superposición de estímulos, Numerosidad, Preescolar.

Responder ordinalmente quer dizer responder sob controle da propriedade relacional "ordem dos estímulos" como explicitaram Miccione, Assis e Costa (2010). Segundo esses autores, "a aprendizagem de um comportamento do tipo ordinal se mostra relevante no contexto da aprendizagem humana devido à sua relação com a aquisição de habilidades matemáticas e linguísticas" (p. 131).

A Análise Experimental do Comportamento tem produzido e sistematizado procedimentos eficazes para o ensino desse tipo de repertório. Os resultados encontrados mostram-se consistentes em explicitar comportamentos relacionados à matemática, como a ordenação, e busca investigar seus determinantes a partir da análise e manipulação de variáveis a ele circunscritas. A utilização dos procedimentos de ensino via encadeamento e sobreposição de estímulos, por exemplo, tem apresentado dados sobre a produção de sequências comportamentais e formação de classes ordinais (para uma revisão, ver Assis, Miccione, \& Nunes, 2010).

Nesse sentido, Green, Stromer e Mackay (1993) propuseram uma análise alternativa do paradigma de equivalência ( $c f$. Sidman, 1994) aplicado com estímulos sequenciais e contribuíram para ampliar aquele modelo de estudo das relações entre estímulos no sentido de observar como organismos podem responder a estímulos em sequência sem que esses estímulos tenham sido relacionados previamente e fora do modelo de emparelhamento com o modelo. Dessa forma, o paradigma de classe ordinal proposto e documentado por esses autores tem sido usado para analisar o efeito do ensino de diferentes sequências comportamentais sobre relações ordinais.

O núcleo dessa proposta é concernente à compreensão da produção de relações ordinais pautada na possibilidade de adequação dos conceitos de cadeia simples e discriminação condicional de desempenhos produzidos por contingências específicas. Os autores defendem que as relações entre os estímulos de uma mesma sequência e entre diferentes sequências podem ser documentadas a partir da realização de testes comportamentais que asseguram as propriedades definidoras de uma relação de ordem: irreflexividade, assimetria, transitividade e conectividade (Stevens, 1951).

Diz-se irreflexiva àquela relação ordinal que não se mostra reflexiva, não podendo um elemento ser seguido por ele mesmo. Nesse caso, a relação $\mathrm{A} 1 \rightarrow \mathrm{A} 1$ não é verdadeira (onde $\rightarrow$ significa vem antes de). A propriedade da assimetria descreve a unidirecionalidade de uma relação. A2 $\rightarrow \mathrm{A} 3$ é uma relação verdadeira, mas o oposto $\mathrm{A} 3 \rightarrow \mathrm{A} 2$ não. $\mathrm{A}$ transitividade é observada quando dois pares de estímulos adjacentes dentro de uma sequência 
são estabelecidos e derivam num terceiro par não adjacente. Uma vez $\mathrm{A} 2 \rightarrow \mathrm{A} 3$ e $\mathrm{A} 3 \rightarrow \mathrm{A} 4$, então A2 $\rightarrow$ A4 é correta, na ausência do A3. Uma relação apresenta a propriedade de conectividade se seus elementos relacionaremse em ordem consistente aos pares em todas as prováveis combinações. Se $\mathrm{A} 1 \rightarrow \mathrm{A} 2 \rightarrow \mathrm{A} 3$, então $\mathrm{A} 1 \rightarrow \mathrm{A} 2, \mathrm{~A} 1 \rightarrow \mathrm{A} 3$ e $\mathrm{A} 2 \rightarrow \mathrm{A} 3$ (e.g., Nunes \& Assis, 2006; Souza, Assis, \& Magalhães, 2005).

Por definição, classes ordinais podem ser formadas a partir do ensino de, pelo menos, duas sequências separadamente. $\mathrm{O}$ fato de a apresentação dos estímulos dessas sequências ocorrer na mesma posição pode facultar a intercambialidade entre eles sob uma nova sequência. A ordem de apresentação dos estímulos torna-se a principal variável de controle sobre a formação das classes de primeiros, segundos, terceiros, etc. por meio de contingências de reforçamento diferencial que demandam respostas de ordená-los numa sequência prevista experimentalmente (e.g., Green et al., 1993; Maydak, Stromer, Mackay, \& Stoddard, 1995; Nunes \& Assis, 2006; Ribeiro, Assis, \& Enumo, 2007; Sigurdardottir, Green, \& Saunders, 1990; Souza, Assis, Magalhães, \& Prado, 2008; Stromer \& Mackay, 1993).

Diversos achados experimentais na literatura (Galy, Camps, \& Melan, 2003; Holcomb, Stromer, \& Mackay, 1997; Maydak et al., 1995; Souza et al., 2005; Stromer \& Mackay, 1993 Experimento 2) mostraram a relevância da nova proposta, na medida em que tornaram possível a produção de sequências e explicitação de fenômenos complexos a partir de contingências tríplices.

Por exemplo, Assis, Corrêa, Souza e Prado (2010) avaliaram a formação de classes ordinais com o ensino de uma única sequência. Participaram cinco crianças na faixa etária de 4 a 6 anos. Os estímulos foram formas abstratas referentes à numerosidade de 1 a 5 . Usou-se um procedimento de ensino por sobreposição de estímulos e, em seguida, testes foram aplicados para avaliar a transitividade e conectividade. Todos os participantes alcançaram o critério de acerto na linha de base. Nos testes de transitividade e conectividade, os cincos participantes responderam prontamente. Além disso, houve generalização para duas novas classes sequenciais. Dos cinco participantes, quatro foram expostos à fase de manutenção, na qual os testes de conectividade foram reaplicados. Três participantes apresentaram um responder consistente com o ensino e um dos participantes respondeu parcialmente. Segundo os autores, o estudo pode ser visto como importante para análise de comportamentos conceituais numéricos, como a contagem e a ordenação. Além disso, a eficácia do procedimento sobre a instalação de uma importante habilidade que pode estar na base do aprendizado de outras habilidades matemáticas mais complexas retrata a economia de tempo envolvida no processo de aquisição de comportamento ordinal emergente.

$\mathrm{O}$ ensino de repertórios sequenciais pode se dar pelo procedimento de sobreposição de pares adjacentes. Nesse tipo de treino, os estímulos são apresentados aos pares e, à medida que um novo estímulo é adicionado na formação de uma nova sequência, o primeiro elemento da sequência anterior é retirado. Dessa forma, os elementos que formam a sequência planejada nunca aparem juntos durante o ensino. Para ensinar a sequência $\mathrm{A} 1 \rightarrow \mathrm{A} 2 \rightarrow \mathrm{A} 3 \rightarrow \mathrm{A} 4 \rightarrow \mathrm{A} 5$, por exemplo, iniciase pelo par $\mathrm{A} 1 \rightarrow \mathrm{A} 2$, em seguida $\mathrm{A} 2 \rightarrow \mathrm{A} 3$, e assim por diante (cf. Holcomb et al., 1997; Souza et al., 2005; Stromer \& Mackay, 1993, estudo 2; Verdu, Souza, \& Lopes Júnior, 2006). Os testes podem envolver estímulos não adjacentes de uma mesma sequência (e.g. $\mathrm{A} 1 \rightarrow \mathrm{A} 5 ; \mathrm{A} 2 \rightarrow \mathrm{A} 4$, etc.) para avaliação da transitividade, ou a substituição de estímulos de sequências diferentes para avaliação da formação de classes (e.g. $\mathrm{A} 1 \rightarrow \mathrm{B} 2 \rightarrow \mathrm{A} 3$ ou $\mathrm{B} 1 \rightarrow \mathrm{A} 3 \rightarrow \mathrm{B} 5)$. Resultados positivos em contingências de testes com sequências de dois ou cinco termos sugerem que o ensino por pares sobrepostos estabelece uma relação de ordem que envolve os pares de estímulos na série ensinada. Esses testes parecem ser mais adequados para a avaliação das propriedades ordinais de assimetria, transitividade e conectividade se comparado ao procedimento de encadeamento onde a configuração dos membros das sequências compartilham semelhanças em contingências de ensino e testes.

Lopes Júnior e Agostini (2004) realizaram dois estudos com o objetivo de verificar a emergência de relações ordinais a partir do ensino de dois pares de estímulos sobrepostos quando um estímulo comum exerce a função de segundo e em seguida de primeiro $(\mathrm{A} 1 \rightarrow \mathrm{A} 2$; 
$\mathrm{A} 2 \rightarrow \mathrm{A} 3)$ na Fase 1; e quando exerce a função de primeiro e em seguida de segundo $(\mathrm{B} 2 \rightarrow \mathrm{B} 1$; $\mathrm{B} 3 \rightarrow \mathrm{B} 2$ ) na fase 2 . A tentativa foi de eliminar o efeito da reversão da ordem com um mesmo conjunto de estímulos. No segundo estudo, o objetivo foi o mesmo, mas a ordem de apresentação dos conjuntos de estímulos era inversa. Dessa forma, o estímulo comum exercia a função de primeiro e em seguida de segundo $(\mathrm{B} 2 \rightarrow \mathrm{B} 1 ; \mathrm{B} 3 \rightarrow \mathrm{B} 2)$; e o inverso na fase $2(\mathrm{~A} 1 \rightarrow \mathrm{A} 2 ; \mathrm{A} 2 \rightarrow \mathrm{A} 3)$. Quatro crianças participaram em cada estudo, sendo que metade não atingiu o critério das primeiras relações da linha de base em ambos os estudos. Duas crianças apresentaram os desempenhos emergentes esperados. $\mathrm{O}$ desempenho das outras duas sugeriu topografias de controle incoerentes em testes com a presença do estímulo comum às relações ensinadas (e.g. $\mathrm{A} 1 \rightarrow \underline{\mathrm{A} 2} \rightarrow \mathrm{A} 3, \mathrm{~B} 3 \rightarrow \underline{\mathrm{B} 2} \rightarrow \mathrm{B} 1)(c f$. Mcllvane \& Dube, 2003). Por exemplo, sempre A1 era apresentado como primeiro e sempre A3 como último (sobre as implicações do uso de apenas dois pares, vide Stromer \& Mackay, 1990). Apesar dessas limitações, os resultados são relevantes para a análise da ordem de apresentação de estímulos no procedimento de sobreposição.

Holcomb et al. (1997) investigaram as variáveis de controle de relações ordinais transitivas a partir da condução de três experimentos em seis crianças. No estudo 1, houve o ensino de duas sequências de seis termos, uma por encadeamento e outra por sobreposição. Os testes ocorreram pela apresentação de pares não adjacentes e, da sequência completa, para quatro crianças. As seis crianças atingiram o critério na linha de base. Nos testes, todas as crianças foram capazes de ordenar três ou mais estímulos sem treino adicional. Apenas uma acertou a sequência completa. No segundo e terceiro experimentos, foi empregado o ensino por sobreposição e testes com reforçamento em três novas configurações. Apenas no Experimento 2 , testes de sequências de três, quatro e cinco termos foram inseridos gradualmente junto aos já descritos no Experimento 1. Dos seis participantes, cinco realizaram a sequência completa e seis apresentaram desempenhos de acordo com a linha de base nas demais configurações dos testes. Já no último Experimento, não houve a introdução gradual de sequências mais longas, sendo apresentadas de forma randômica. Quatro de cinco participantes atingiram o critério da linha de base e apresentaram um índice alto de acerto nos testes. O experimento 3 foi uma replicação do 2 acrescido de uma entrevista após os testes, as quais avaliaram a mediação do comportamento verbal nos desempenhos apresentados. Os resultados sugerem que a transitividade pode ocorrer mesmo na ausência da mediação verbal e a inferência transitiva baseia-se no fato de que as sequências de pares não adjacentes emergem sem treino direto. Tal estudo tem relevância para as considerações implicadas no procedimento utilizado com a mesma população deste estudo.

Mais recentemente, alguns autores (Verdu et al., 2006) replicaram Stromer e Mackay (1993) e Holcomb et al. (1997), tendo como participantes cinco crianças do ensino fundamental com dificuldade de acompanhar o currículo escolar do primeiro ciclo (ensino fundamental). Os autores utilizaram o procedimento de sobreposição de estímulos (overlapping two-stimuli sequences), com cinco estímulos do conjunto "A" (e.g. A1 $\rightarrow \mathrm{A} 2$, $\mathrm{A} 2 \rightarrow \mathrm{A} 3, \quad \mathrm{~A} 3 \rightarrow \mathrm{A} 4, \quad \mathrm{~A} 4 \rightarrow \mathrm{A} 5)$ e cinco do conjunto "B", com formas abstratas apresentadas aos pares. Os testes consistiram na verificação da emergência de sequências de três, quatro e cinco termos, dos respectivos conjuntos separadamente. Três participantes apresentaram um responder consistente com a linha de base nos testes de substituição entre estímulos dos conjuntos "A" e "B", indicando a formação de classes de estímulos que ocupam a mesma posição ordinal.

Se, por um lado, nos estudos que tratam de relações ordinais em contingências simples, há a reversão de função ordinal do membro comum aos pares, nos estudos sobre controle condicional de relações ordinais há uma variável adicional: a reversão da função da ordem dos estímulos de um mesmo par. Tal variável pode gerar dificuldades no estabelecimento do controle condicional. Por exemplo, na presença do estímulo $\mathrm{X} 1$, podemse ensinar duas relações em que o estímulo comum (A2) exerça as funções de segundo na primeira relação $(\mathrm{A} 1 \rightarrow \mathrm{A} 2)$ e de primeiro na relação seguinte $(\mathrm{A} 2 \rightarrow \mathrm{A} 3)$. Por outro lado, na presença de $\mathrm{X} 2$, o mesmo estímulo A2 pode exercer a função inicialmente de primeiro $(\mathrm{A} 2 \rightarrow \mathrm{A} 1)$ e em seguida de segundo $(\mathrm{A} 3 \rightarrow \mathrm{A} 2)$. Trata-se de uma importante variável a ser considerada durante a análise dos dados. 
Os estudos descritos até aqui sugerem a dificuldade existente no procedimento de ensino por sobreposição com pares de estímulos devido à reversão da função, seja sob controle simples ou condicional (e.g. A1 $\rightarrow$ A2; A2 $\rightarrow$ A3). Portanto, o objetivo deste estudo foi replicar sistematicamente o procedimento de ensino por sobreposição de estímulos numéricos com três membros, expandindo o número de classes formadas. Para isso, um novo conjunto de estímulos foi introduzido (formas não representacionais), possibilitando inferir relações de substituibilidade (conectividade), caracterizando a formação de classes ordinais. Além disso, possibilita o avanço teórico e metodológico na medida em que utiliza o procedimento de ensino de trios de estímulos sobrepostos.

\section{Metodologia}

\section{Participantes}

Participaram do estudo três pré-escolares: LEX com 5 anos e cinco meses, gênero masculino; PRI com 5 anos e dez meses, gênero feminino e VAN com 5 anos e nove meses, gênero masculino. Todos de família de baixa renda. A seleção teve como base o desempenho apresentado nos pré-testes. Os responsáveis pelos participantes assinaram um termo de consentimento livre e esclarecido, autorizando a participação do aluno no estudo, conforme exigência da Resolução 196/96 do Conselho Nacional de Saúde, aprovado pelo comitê de ética da Universidade (Protocolo $\mathrm{n}^{\circ}$ 095/09 - CEP-ICS/UFPA).

\section{Ambiente experimental e equipamento}

As sessões experimentais foram realizadas em uma sala de leitura de uma instituição de assistência social, localizada na periferia da cidade de Belém. A sala apresentava um relativo isolamento acústico, iluminada e climatizada. $\mathrm{O}$ participante permanecia sentado frente a um microcomputador, com a experimentadora ao seu lado, monitorando a sessão experimental.

Um microcomputador, modelo IBM PENTIUM de $300 \mathrm{MHz}$, fornecia suporte ao estudo. O programa REL (versão 6.1 for Windows - Santos, Silva, Baptista, \& Assis, 1997) elaborado em linguagem Java, desenvolvido especialmente para esta pesquisa, apresentava os estímulos e registrava o número de tentativas, as posições de cada estímulo na tela e os estímulos selecionados pelo participante.

\section{Estímulos}

Foram utilizados dois conjuntos de estímulos (A e B). O primeiro continha os numerais cardinais de 1 a 9 . O segundo era composto por uma forma não representacional em nove quantidades, variando em duas dimensões: disposição espacial e tamanho. Os estímulos dos conjuntos estão identificados de forma alfanumérica nas Figuras 1 e 2, respectivamente. Onde as letras A e B indicam os Conjuntos e os numerais, a posição do estímulo na sequência.

\section{Procedimento}

O procedimento foi dividido em pré-teste, ensino e testes (ver Tabela 1).

\section{Pré-testes}

Os três participantes (LEX, PRI e VAN) foram submetidos às duas subfases da fase de pré-teste:

\section{Pré-teste de nomeação}

Avaliou-se a nomeação dos estímulos a serem utilizados no ensino. Os numerais do conjunto "A" foram organizados no programa PowerPoint de forma aleatória com a apresentação de todos os estímulos três vezes consecutivas. Foi dito ao participante: "Aparecerá uma figura na tela e você deverá dizer o nome dela". As respostas foram anotadas em uma folha de registro pela pesquisadora e serviram de base para a seleção dos participantes. Em seguida, era apresentado aos participantes um jogo intitulado Bloco lógico de marca FUNBEC contendo 48 peças de madeira em quatro dimensões: cores (azul, vermelho e amarelo); formas (triângulo, quadrado e círculo); tamanho (pequeno, médio e grande) e espessura (grossa e fina). As peças eram dispostas sobre uma mesa aleatoriamente. A experimentadora solicitava ao participante que selecionasse cinco, três ou sete peças, por exemplo. Sendo uma tentativa com cada uma das nove quantidades. Participaram do estudo os alunos que não nomearam corretamente, ou nomearam até $20 \%$ das figuras nos testes. 


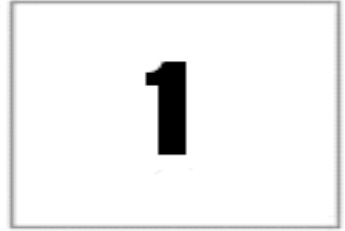

A1

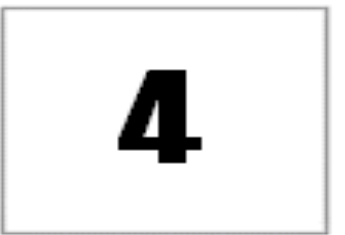

A4

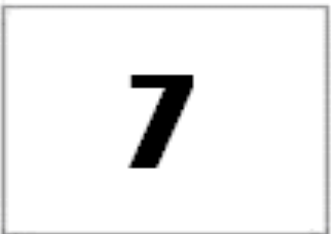

A 7

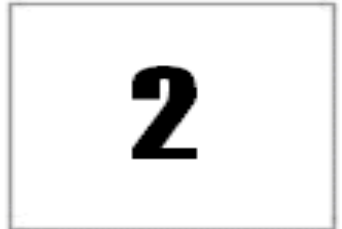

A2

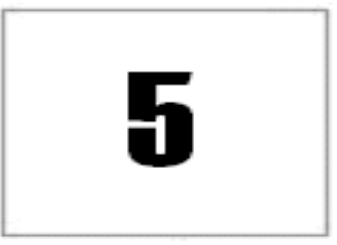

A5

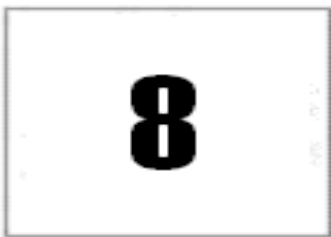

A8

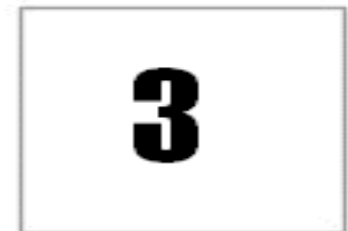

A3
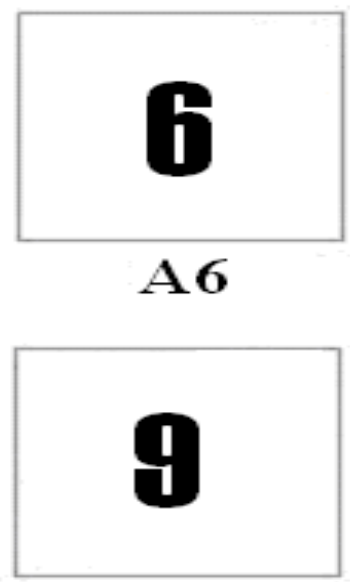

A9

Figura 1 - Estímulos do Conjunto A usados no estudo.

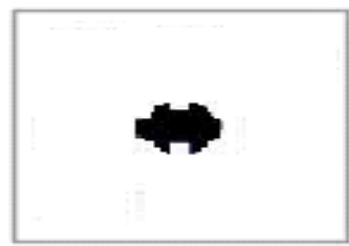

B 1

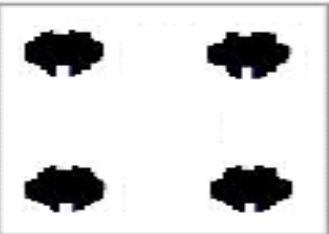

B4

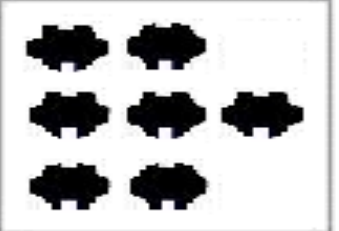

B7

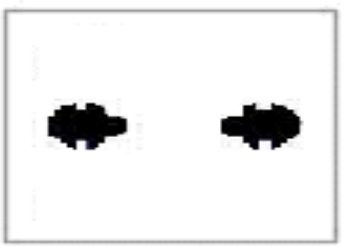

B2

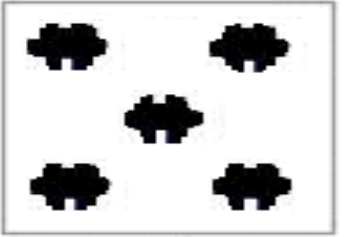

B5

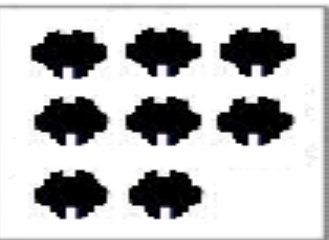

B8

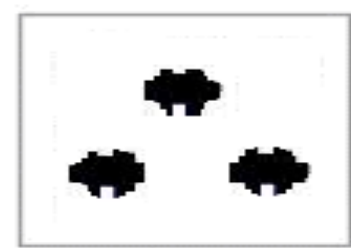

B3

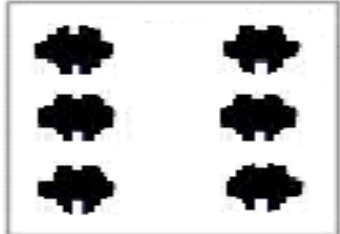

B6

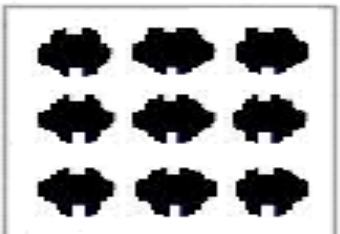

B9

Figura 2 - Estímulos do Conjunto B usados no estudo. 
Tabela 1 - Sumário do procedimento de ensino, sondas e testes com fases, tipo de bloco e tipo de tentativas.

\begin{tabular}{|c|c|c|}
\hline Fase & Tipo de bloco & Tipo de tentativa(s) \\
\hline 1 & Pré-testes & $\begin{array}{l}\text { Nomeação } \\
\text { Ordenação } \\
\end{array}$ \\
\hline 2 & Levantamento de Preferências & \\
\hline \multirow[t]{15}{*}{3 e 4} & $\begin{array}{l}\text { Relações ensinadas e sondas por trios } \\
\text { sobrepostos } \\
\text { Conjunto } \mathrm{A}\end{array}$ & \\
\hline & Ensino & $\mathrm{A} 1 \rightarrow \mathrm{A} 2 \rightarrow \mathrm{A} 3$ \\
\hline & Sonda & $\mathrm{A} 1 \rightarrow \mathrm{A} 2 \rightarrow \mathrm{A} 3$ \\
\hline & Ensino & $\mathrm{A} 3 \rightarrow \mathrm{A} 4 \rightarrow \mathrm{A} 5$ \\
\hline & Sonda & $\mathrm{A} 3 \rightarrow \mathrm{A} 4 \rightarrow \mathrm{A} 5$ \\
\hline & Ensino & $\mathrm{A} 1 \rightarrow \mathrm{A} 2 \rightarrow \mathrm{A} 3, \mathrm{~A} 3 \rightarrow \mathrm{A} 4 \rightarrow \mathrm{A} 5$ \\
\hline & Sonda & $\mathrm{A} 1 \rightarrow \mathrm{A} 2 \rightarrow \mathrm{A} 3, \mathrm{~A} 3 \rightarrow \mathrm{A} 4 \rightarrow \mathrm{A} 5$ \\
\hline & Ensino & A5 $\rightarrow \mathrm{A} 6 \rightarrow \mathrm{A} 7$ \\
\hline & Sonda & $A 5 \rightarrow A 6 \rightarrow A 7$ \\
\hline & Ensino & $\mathrm{A} 1 \rightarrow \mathrm{A} 2 \rightarrow \mathrm{A} 3, \mathrm{~A} 3 \rightarrow \mathrm{A} 4 \rightarrow \mathrm{A} 5, \mathrm{~A} 5 \rightarrow \mathrm{A} 6 \rightarrow \mathrm{A} 7$ \\
\hline & Sonda & $\mathrm{A} 1 \rightarrow \mathrm{A} 2 \rightarrow \mathrm{A} 3, \mathrm{~A} 3 \rightarrow \mathrm{A} 4 \rightarrow \mathrm{A} 5, \mathrm{~A} 5 \rightarrow \mathrm{A} 6 \rightarrow \mathrm{A} 7$ \\
\hline & $\overline{E \text { Ensino }}$ & A7 $\rightarrow \mathrm{A} 8 \rightarrow \mathrm{A} 9$ \\
\hline & Sonda & $A 7 \rightarrow A 8 \rightarrow A 9$ \\
\hline & Ensino & $\mathrm{A} 1 \rightarrow \mathrm{A} 2 \rightarrow \mathrm{A} 3, \mathrm{~A} 3 \rightarrow \mathrm{A} 4 \rightarrow \mathrm{A} 5, \mathrm{~A} 5 \rightarrow \mathrm{A} 6 \rightarrow \mathrm{A} 7, \mathrm{~A} 7 \rightarrow \mathrm{A} 8 \rightarrow \mathrm{A} 9$ \\
\hline & Sonda & $\mathrm{A} 1 \rightarrow \mathrm{A} 2 \rightarrow \mathrm{A} 3, \mathrm{~A} 3 \rightarrow \mathrm{A} 4 \rightarrow \mathrm{A} 5, \mathrm{~A} 5 \rightarrow \mathrm{A} 6 \rightarrow \mathrm{A} 7, \mathrm{~A} 7 \rightarrow \mathrm{A} 8 \rightarrow \mathrm{A} 9$ \\
\hline 5 & $\begin{array}{l}\text { Teste de transitividade } \\
\text { Conjunto } \mathrm{A}\end{array}$ & $\begin{array}{l}\mathrm{A} 1 \rightarrow \mathrm{A} 3 \rightarrow \mathrm{A} 5 \\
\mathrm{~A} 3 \rightarrow \mathrm{A} 5 \rightarrow \mathrm{A} 7 \\
\mathrm{~A} 5 \rightarrow \mathrm{A} 7 \rightarrow \mathrm{A} 9\end{array}$ \\
\hline \multirow[t]{15}{*}{6 e 7} & $\begin{array}{c}\text { Relações ensinadas e sondas por trios } \\
\text { sobrepostos } \\
\text { Conjunto B } \\
\end{array}$ & \\
\hline & Ensino & $\mathrm{B} 1 \rightarrow \mathrm{B} 2 \rightarrow \mathrm{B} 3$ \\
\hline & Sonda & $\mathrm{B} 1 \rightarrow \mathrm{B} 2 \rightarrow \mathrm{B} 3$ \\
\hline & Ensino & $\mathrm{B} 3 \rightarrow \mathrm{B} 4 \rightarrow \mathrm{B} 5$ \\
\hline & Sonda & $\mathrm{B} 3 \rightarrow \mathrm{B} 4 \rightarrow \mathrm{B} 5$ \\
\hline & Ensino & $\mathrm{B} 1 \rightarrow \mathrm{B} 2 \rightarrow \mathrm{B} 3, \mathrm{~B} 3 \rightarrow \mathrm{B} 4 \rightarrow \mathrm{B} 5$ \\
\hline & Sonda & $\mathrm{B} 1 \rightarrow \mathrm{B} 2 \rightarrow \mathrm{B} 3, \mathrm{~B} 3 \rightarrow \mathrm{B} 4 \rightarrow \mathrm{B} 5$ \\
\hline & $\overline{E \text { Ensino }}$ & B $\mathrm{B} 5 \rightarrow \mathrm{B} 6 \rightarrow \mathrm{B} 7$ \\
\hline & Sonda & $\mathrm{B} 5 \rightarrow \mathrm{B} 6 \rightarrow \mathrm{B} 7$ \\
\hline & Ensino & $\mathrm{B} 1 \rightarrow \mathrm{B} 2 \rightarrow \mathrm{B} 3, \mathrm{~B} 3 \rightarrow \mathrm{B} 4 \rightarrow \mathrm{B} 5, \mathrm{~B} 5 \rightarrow \mathrm{B} 6 \rightarrow \mathrm{B} 7$ \\
\hline & Sonda & $\mathrm{B} 1 \rightarrow \mathrm{B} 2 \rightarrow \mathrm{B} 3, \mathrm{~B} 3 \rightarrow \mathrm{B} 4 \rightarrow \mathrm{B} 5, \mathrm{~B} 5 \rightarrow \mathrm{B} 6 \rightarrow \mathrm{B} 7$ \\
\hline & Ensino & $\mathrm{B} 7 \rightarrow \mathrm{B} 8 \rightarrow \mathrm{B} 9$ \\
\hline & Sonda & $\mathrm{B} 7 \rightarrow \mathrm{B} 8 \rightarrow \mathrm{B} 9$ \\
\hline & $\overline{\text { Ensino }}$ & $\overline{\mathrm{B} 1 \rightarrow \mathrm{B} 2 \rightarrow \mathrm{B} 3, \mathrm{~B} 3 \rightarrow \mathrm{B} 4 \rightarrow \mathrm{B} 5, \mathrm{~B} 5 \rightarrow \mathrm{B} 6 \rightarrow \mathrm{B} 7, \mathrm{~B} 7 \rightarrow \mathrm{B} 8 \rightarrow \mathrm{B} 9}$ \\
\hline & Sonda & $\overline{\mathrm{B} 1 \rightarrow \mathrm{B} 2 \rightarrow \mathrm{B} 3, \mathrm{~B} 3 \rightarrow \mathrm{B} 4 \rightarrow \mathrm{B} 5, \mathrm{~B} 5 \rightarrow \mathrm{B} 6 \rightarrow \mathrm{B} 7, \mathrm{~B} 7 \rightarrow \mathrm{B} 8 \rightarrow \mathrm{B} 9}$ \\
\hline 8 & $\begin{array}{l}\text { Teste de transitividade } \\
\text { Conjunto B }\end{array}$ & $\begin{array}{l}\mathrm{B} 1 \rightarrow \mathrm{B} 3 \rightarrow \mathrm{B} 5 \\
\mathrm{~B} 3 \rightarrow \mathrm{B} 5 \rightarrow \mathrm{B} 7 \\
\mathrm{~B} 5 \rightarrow \mathrm{B} 7 \rightarrow \mathrm{B} 9\end{array}$ \\
\hline \multirow[t]{2}{*}{9} & Teste de conectividade A/B & $\begin{array}{l}\mathrm{A} 1 \rightarrow \mathrm{B} 2 \rightarrow \mathrm{A} 3 \\
\mathrm{~B} 4 \rightarrow \mathrm{A} 5 \rightarrow \mathrm{B} 6 \\
\mathrm{~A} 7 \rightarrow \mathrm{B} 8 \rightarrow \mathrm{A} 9\end{array}$ \\
\hline & & $\begin{array}{l}\mathrm{B} 1 \rightarrow \mathrm{A} 2 \rightarrow \mathrm{B} 3 \\
\mathrm{~A} 4 \rightarrow \mathrm{B} 5 \rightarrow \mathrm{A} 6 \\
\mathrm{~B} 7 \rightarrow \mathrm{A} 8 \rightarrow \mathrm{B} 9\end{array}$ \\
\hline 10 & $\begin{array}{l}\text { Teste de Generalização } \\
\text { Conjunto A } \\
\text { Conjunto B }\end{array}$ & $\begin{array}{l}\mathrm{A} 1 \rightarrow \mathrm{A} 2 \rightarrow \mathrm{A} 3 \rightarrow \mathrm{A} 4 \rightarrow \mathrm{A} 5 \rightarrow \mathrm{A} 6 \rightarrow \mathrm{A} 7 \rightarrow \mathrm{A} 8 \rightarrow \mathrm{A} 9 \\
\mathrm{~B} 1 \rightarrow \mathrm{B} 2 \rightarrow \mathrm{B} 3 \rightarrow \mathrm{B} 4 \rightarrow \mathrm{B} 5 \rightarrow \mathrm{B} 6 \rightarrow \mathrm{B} 7 \rightarrow \mathrm{B} 8 \rightarrow \mathrm{B} 9\end{array}$ \\
\hline
\end{tabular}




\section{Pré-teste de ordinalidade}

Após ser exposto ao pré-teste de ordenação, avaliou-se o desempenho de ordenação de figuras de uma sequência lógica de ações. Para tanto, um jogo (marca FUNBEC) contendo figuras de encaixe em madeira foi utilizado. O participante recebia uma única instrução: "ordene as figuras". Na presença de cada uma das quatro sequências lógicas, o participante tinha três oportunidades para encaixar as peças de modo a formar uma única possibilidade de sequência (por exemplo, a cena de uma pessoa atravessando a rua subdividida em cinco partes). Portanto, em 12 tentativas, deveria, pelo menos, acertar 4 sequências.

O objetivo dos pré-testes foi verificar a presença de repertórios envolvidos naqueles observados durante $o$ procedimento programado.

\section{Levantamento de preferências}

Nessa etapa, antes de iniciar cada sessão experimental realizou-se um levantamento de preferências de cada participante (por exemplo, jogos, pipoca e brindes diversos). Perguntavase ao participante o que ele(ela) gostaria de receber pela sua participação no final das atividades. $\mathrm{O}$ objetivo desta fase foi organizar, para cada participante, estímulos reforçadores a serem manipulados durante o procedimento experimental.

\section{Ensino por sobreposição}

O participante era exposto ao procedimento de ensino de ordenação com três estímulos apresentados simultaneamente na área de escolha. Cada tentativa iniciava com um quadrado em branco, na parte superior da tela e com a instrução: "Toque no quadrado". Ao tocar o quadrado, os primeiros estímulos (A1, A2 e A3) eram apresentados simultaneamente em três "células" das oito previstas na área de escolha, na parte inferior da tela em posições aleatórias. A pesquisadora fornecia as seguintes instruções ao participante: "Quando aparecerem as figuras, toque em uma, depois em outra e logo em outra". O participante deveria tocar primeiro em A1, depois em A2 e por último, em A3. Ao tocar A1, o mesmo deslocava-se para a área de construção. Ao tocar A2, o segundo estímulo também se deslocava para a área de construção, permanecendo ao lado direito de A1. E, ao tocar em A3, o terceiro e último estímulo se deslocava da mesma forma, posicionando-se ao lado direito de A2. Após completar a sequência de respostas corretas, uma animação gráfica era apresentada na tela do computador. Simultaneamente, uma consequência social (e.g. "Muito bem!", "Parabéns!") era fornecido pela pesquisadora e um vídeo com desenho animado de $5 \mathrm{~s}$ de duração era apresentado. $\mathrm{O}$ critério de acerto para avançar para a próxima sequência era responder corretamente ao trio por três vezes consecutivas, sem erro. Sequências de respostas diferentes das sequências programadas eram seguidas por escurecimento da tela por $3 \mathrm{~s}$ (e.g. $\mathrm{A} 2 \rightarrow \mathrm{A} 1 \rightarrow \mathrm{A} 3)$ e a mesma configuração de estímulos era reapresentada na "área de escolha" na mesma posição (procedimento de correção). O participante era exposto a um mesmo trio até 10 vezes, no máximo. Em seguida, havia a apresentação da mesma configuração de estímulos na tela (sonda), mas, não havia consequências ao término da sequência. Após alcançar o critério de acerto, um novo trio era apresentado, mantendo-se a última figura do trio anterior (e.g. A3 $\rightarrow$ A4 $\rightarrow$ A5). Após alcançar o mesmo critério de ensino da sequência anterior com a nova sequência, um bloco de ensino de cada relação ensinada era apresentado duas vezes, e assim por diante, até completar a última sequência $(\mathrm{A} 7 \rightarrow \mathrm{A} 8 \rightarrow \mathrm{A} 9$ - ver sumário na Tabela 1$)$. Um bloco de sonda com cada sequência anterior era apresentado, após cada sequência ensinada. $\mathrm{O}$ objetivo da fase de ensino foi estabelecer uma linha de base para analisar o desempenho nos testes seguintes.

\section{Sonda}

Após cada trio ensinado por sobreposição, foi introduzida uma tentativa de sonda similar à tentativa de ensino, mas sem reforçamento diferencial. Por exemplo, após o ensino do trio $\mathrm{A} 1 \rightarrow \mathrm{A} 2 \rightarrow \mathrm{A} 3$, havia uma sonda $\mathrm{A} 1 \rightarrow \mathrm{A} 2 \rightarrow \mathrm{A} 3$; após o ensino A3 $\rightarrow \mathrm{A} 4 \rightarrow \mathrm{A} 5$, havia uma sonda $\mathrm{A} 3 \rightarrow \mathrm{A} 4 \rightarrow \mathrm{A} 5$, e assim por diante, até completar a última sequência $(\mathrm{A} 7 \rightarrow \mathrm{A} 8 \rightarrow \mathrm{A} 9)$. Caso $O$ participante respondesse diferentemente, era reapresentado o mesmo bloco de ensino. A instrução era: "Quando aparecerem as figuras, toque em uma figura, depois em outra e logo em outra. Dessa vez não aparecerá o Mickey." Os parâmetros eram os mesmos para exposição aos estímulos do conjunto "B". O objetivo era introduzir aos participantes a ocorrência de tentativas sem reforçamento diferencial, da mesma forma como nas tentativas de testes. 


\section{Teste de transitividade}

$O$ teste de transitividade consistia na apresentação de três sequências com três estímulos não adjacentes que não haviam sido apresentados na fase de ensino: $\mathrm{A} 1 \rightarrow \mathrm{A} 3 \rightarrow \mathrm{A} 5$; $\mathrm{A} 3 \rightarrow \mathrm{A} 5 \rightarrow \mathrm{A} 7 ; \mathrm{A} 5 \rightarrow \mathrm{A} 7 \rightarrow \mathrm{A} 9$ ou $\mathrm{B} 1 \rightarrow \mathrm{B} 3 \rightarrow \mathrm{B} 5$; $\mathrm{B} 3 \rightarrow \mathrm{B} 5 \rightarrow \mathrm{B} 7$; $\mathrm{B} 5 \rightarrow \mathrm{B} 7 \rightarrow \mathrm{B} 9$. O objetivo era verificar se tais relações emergiriam sem qualquer treino adicional.

\section{Teste de conectividade ou} substituibilidade dos Conjuntos A/B

Este teste avaliava a formação de classes de estímulos ordinais decorrentes do treino original a partir da apresentação de seis sequências não apresentadas na fase de ensino. As sequências apresentadas foram:

$$
\begin{aligned}
& \mathrm{A} 1 \rightarrow \mathrm{B} 2 \rightarrow \mathrm{A} 3 \\
& \mathrm{~B} 4 \rightarrow \mathrm{A} 5 \rightarrow \mathrm{B} 6 \\
& \mathrm{~A} 7 \rightarrow \mathrm{B} 8 \rightarrow \mathrm{A} 9 \\
& \mathrm{~B} 1 \rightarrow \mathrm{A} 2 \rightarrow \mathrm{B} 3 \\
& \mathrm{~A} 4 \rightarrow \mathrm{B} 5 \rightarrow \mathrm{A} 6 \\
& \mathrm{~B} 7 \rightarrow \mathrm{A} 8 \rightarrow \mathrm{B} 9
\end{aligned}
$$

\section{Testes de Generalização}

Os participantes deveriam ordenar da esquerda para a direita as peças de EVA contendo numerais de 1 a 9. Em seguida, deveria ordenar as figuras de uma sequência lógica do mesmo jogo confeccionado pela FUNBEC e usado no pré-teste, contendo peças em madeira com figuras para encaixar e completar uma ação. A única instrução fornecida ao participante era: "Faça o mesmo que no joguinho do computador: coloque uma figura depois da outra". O objetivo deste teste final era verificar se haveria transferência de funções discriminativas de numerosidade e ordinais do ambiente informatizado no laboratório para um ambiente com material escolar.

\section{Resultados}

\section{Pré-teste}

No pré-teste de nomeação, verificou-se que os participantes não nomearam a maioria dos numerais cardinais de 1 a 9. Dois participantes (LEX e PRI) nomearam corretamente $33,33 \%$ aos numerais, em cada uma das três tentativas consecutivas previstas. $\mathrm{O}$ participante VAN respondeu corretamente $44,44 \%$ aos numerais, nas três tentativas consecutivas previstas.
No pré-teste de ordinalidade, nenhum participante ordenou as quatro sequências lógicas programadas.

\section{Ensino por sobreposição}

Os participantes concluíram o ensino por sobreposição. Considerando o ensino dos quatro trios do conjunto $\mathrm{A}(\mathrm{A} 1 \rightarrow \mathrm{A} 2 \rightarrow \mathrm{A} 3$, $\mathrm{A} 3 \rightarrow \mathrm{A} 4 \rightarrow \mathrm{A} 5, \mathrm{~A} 5 \rightarrow \mathrm{A} 6 \rightarrow \mathrm{A} 7$ e $\mathrm{A} 7 \rightarrow \mathrm{A} 8 \rightarrow \mathrm{A} 9)$, o participante LEX obteve uma porcentagem de acerto imediato de $25 \%$ (1/4). Entende-se por acerto imediato os acertos na primeira tentativa de cada trio, isto é, a primeira vez que o participante é exposto a cada trio. No bloco de ensino inicial de cada trio (critério de três corretas consecutivas cada), o participante LEX obteve: $75 \%$ de acerto no ensino dos trios $\mathrm{A} 1 \rightarrow \mathrm{A} 2 \rightarrow \mathrm{A} 3$ e $\mathrm{A} 3 \rightarrow \mathrm{A} 4 \rightarrow \mathrm{A} 5(3 / 4)$ e $80 \%$ de acerto no ensino do trio e A5 $\rightarrow \mathrm{A} 6 \rightarrow \mathrm{A} 7$, acertando $4 / 5$, no último trio $(\mathrm{A} 7 \rightarrow \mathrm{A} 8 \rightarrow \mathrm{A} 9)$ obteve $60 \%$ de acerto, o que corresponde a $3 / 5$ de acerto. O participante PRI obteve uma porcentagem de acerto imediato de $0 \%(0 / 4)$ e no ensino inicial de cada trio obteve: $75 \%$ de acerto (3/4) no ensino dos trios $\mathrm{A} 1 \rightarrow \mathrm{A} 2 \rightarrow \mathrm{A} 3$, $\mathrm{A} 5 \rightarrow \mathrm{A} 6 \rightarrow \mathrm{A} 7$ e $\mathrm{A} 7 \rightarrow \mathrm{A} 8 \rightarrow \mathrm{A} 9$ e $50 \%$ no trio $\mathrm{A} 3 \rightarrow \mathrm{A} 4 \rightarrow \mathrm{A} 5$ (3/6). O participante VAN obteve uma porcentagem de acerto imediato de $25 \%(1 / 4)$ e no ensino inicial de cada trio obteve: $60 \%$ de acerto (3/5) no ensino do trio $\mathrm{A} 1 \rightarrow \mathrm{A} 2 \rightarrow \mathrm{A} 3,75 \%$ nos trios $\mathrm{A} 3 \rightarrow \mathrm{A} 4 \rightarrow \mathrm{A} 5$ e $\mathrm{A} 5 \rightarrow \mathrm{A} 6 \rightarrow \mathrm{A} 7 \quad(3 / 4)$ e $100 \%$ no trio $\mathrm{A} 7 \rightarrow \mathrm{A} 8 \rightarrow \mathrm{A} 9$ (3/3). As Tabelas 2,4 e 6 apresentam esses dados.

Com relação ao ensino dos quatro trios do conjunto $\mathrm{B} \quad(\mathrm{B} 1 \rightarrow \mathrm{B} 2 \rightarrow \mathrm{B} 3, \quad \mathrm{~B} 3 \rightarrow \mathrm{B} 4 \rightarrow \mathrm{B} 5$, $\mathrm{B} 5 \rightarrow \mathrm{B} 6 \rightarrow \mathrm{B} 7$ e $\mathrm{B} 7 \rightarrow \mathrm{B} 8 \rightarrow \mathrm{B} 9$ ), o participante LEX obteve uma porcentagem de acerto imediato de $100 \%$ (4/4). Também obteve $100 \%$ de acerto (3/3) nos trios: $\mathrm{B} 1 \rightarrow \mathrm{B} 2 \rightarrow \mathrm{B} 3$ e $\mathrm{B} 5 \rightarrow \mathrm{B} 6 \rightarrow \mathrm{B} 7$. Nos trios $\mathrm{B} 3 \rightarrow \mathrm{B} 4 \rightarrow \mathrm{B} 5$ e $\mathrm{B} 7 \rightarrow \mathrm{B} 8 \rightarrow \mathrm{B} 9$, o participante LEX obteve $80 \%$ de acerto, acertando 4/5. Da mesma forma, o participante PRI alcançou $100 \%$ de acerto imediato e $100 \%$ de acerto (3/3) nos trios: $\mathrm{B} 1 \rightarrow \mathrm{B} 2 \rightarrow \mathrm{B} 3, \mathrm{~B} 3 \rightarrow \mathrm{B} 4 \rightarrow \mathrm{B} 5$ e $\mathrm{B} 7 \rightarrow \mathrm{B} 8 \rightarrow \mathrm{B} 9$. No trio $\mathrm{B} 5 \rightarrow \mathrm{B} 6 \rightarrow \mathrm{B} 7$, o participante PRI obteve $83 \%$ (5/6). Já o participante VAN obteve $75 \%$ de acerto inicial (3/4). Nos trios $\mathrm{B} 1 \rightarrow \mathrm{B} 2 \rightarrow \mathrm{B} 3$ e $\mathrm{B} 7 \rightarrow \mathrm{B} 8 \rightarrow \mathrm{B} 9$, alcançou $100 \%$ de acerto $(3 / 3)$. E nos trios $\mathrm{B} 3 \rightarrow \mathrm{B} 4 \rightarrow \mathrm{B} 5$ e B5 $\rightarrow \mathrm{B} 6 \rightarrow \mathrm{B} 7$, obteve $75 \%$ e $83 \%$ de acerto (3/4 e 5/6), respectivamente. Esses dados podem ser observados nas Tabelas 3, 5 e 7 . 
Tabela 2 - Primeiro ensino e sonda de cada trio de LEX do Conjunto A.

\begin{tabular}{cccccc}
\hline $\begin{array}{c}\text { Tipo de bloco na } \\
\text { ordem de } \\
\text { apresentação }\end{array}$ & $\begin{array}{c}\text { Número de } \\
\text { apresentação do } \\
\text { bloco na sessão }\end{array}$ & $\begin{array}{c}\text { Tipo de tentativa } \\
\text { na ordem de } \\
\text { apresentação }\end{array}$ & $\begin{array}{c}\text { Resposta por } \\
\text { tentativa na } \\
\text { ordem de } \\
\text { apresentação }\end{array}$ & $\begin{array}{c}\text { Número de } \\
\text { acertos } \\
\text { sobre } 0 \\
\text { número de } \\
\text { tentativas }\end{array}$ & $\begin{array}{c}\text { Atingiu o } \\
\text { critério de } \\
\text { acerto }\end{array}$ \\
\hline Ensino & 1 & $\mathrm{~A} 1 \rightarrow \mathrm{A} 2 \rightarrow \mathrm{A} 3$ & $0+++$ & $3 / 4$ & $\mathrm{~S}$ \\
Sonda & 1 & $\mathrm{~A} 1 \rightarrow \mathrm{A} 2 \rightarrow \mathrm{A} 3$ & + & $1 / 1$ & $\mathrm{~S}$ \\
Ensino & 1 & $\mathrm{~A} 3 \rightarrow \mathrm{A} 4 \rightarrow \mathrm{A} 5$ & $0+++$ & $3 / 4$ & $\mathrm{~S}$ \\
Sonda & 1 & $\mathrm{~A} 3 \rightarrow \mathrm{A} 4 \rightarrow \mathrm{A} 5$ & + & $1 / 1$ & $\mathrm{~S}$ \\
Ensino & 1 & $\mathrm{~A} 5 \rightarrow \mathrm{A} 6 \rightarrow \mathrm{A} 7$ & $+0+++$ & $4 / 5$ & $\mathrm{~S}$ \\
Sonda & 1 & $\mathrm{~A} 5 \rightarrow \mathrm{A} 6 \rightarrow \mathrm{A} 7$ & + & $1 / 1$ & $\mathrm{~S}$ \\
Ensino & 1 & $\mathrm{~A} 7 \rightarrow \mathrm{A} 8 \rightarrow \mathrm{A} 9$ & $00+++$ & $3 / 5$ & $\mathrm{~S}$ \\
Sonda & 1 & $\mathrm{~A} 7 \rightarrow \mathrm{A} 8 \rightarrow \mathrm{A} 9$ & + & $1 / 1$ & $\mathrm{~S}$ \\
\hline
\end{tabular}

Nota. O sinal + corresponde ao acerto e o sinal 0 corresponde a outro tipo de responder sequencial.

Tabela 3 - Primeiro ensino e sonda de cada trio de LEX do Conjunto $B$.

\begin{tabular}{cccccc}
\hline $\begin{array}{c}\text { Tipo de bloco na } \\
\text { ordem de } \\
\text { apresentação }\end{array}$ & $\begin{array}{c}\text { Número de } \\
\text { apresentação do } \\
\text { bloco na sessão }\end{array}$ & $\begin{array}{c}\text { Tipo de tentativa } \\
\text { na ordem de } \\
\text { apresentação }\end{array}$ & $\begin{array}{c}\text { Resposta por } \\
\text { tentativa na } \\
\text { ordem de } \\
\text { apresentação }\end{array}$ & $\begin{array}{c}\text { Número de } \\
\text { acertos } \\
\text { sobre o } \\
\text { número de } \\
\text { tentativas }\end{array}$ & $\begin{array}{c}\text { Atingiu o } \\
\text { critério de } \\
\text { acerto }\end{array}$ \\
\hline Ensino & 1 & $\mathrm{~B} 1 \rightarrow \mathrm{B} 2 \rightarrow \mathrm{B} 3$ & +++ & $3 / 3$ & $\mathrm{~S}$ \\
Sonda & 1 & $\mathrm{~B} 1 \rightarrow \mathrm{B} 2 \rightarrow \mathrm{B} 3$ & + & $1 / 1$ & $\mathrm{~S}$ \\
Ensino & 1 & $\mathrm{~B} 3 \rightarrow \mathrm{B} 4 \rightarrow \mathrm{B} 5$ & $+0+++$ & $4 / 5$ & $\mathrm{~S}$ \\
Sonda & 1 & $\mathrm{~B} 3 \rightarrow \mathrm{B} 4 \rightarrow \mathrm{B} 5$ & + & $1 / 1$ & $\mathrm{~S}$ \\
Ensino & 1 & $\mathrm{~B} 5 \rightarrow \mathrm{B} 6 \rightarrow \mathrm{B} 7$ & +++ & $3 / 3$ & $\mathrm{~S}$ \\
Sonda & 1 & $\mathrm{~B} 5 \rightarrow \mathrm{B} 6 \rightarrow \mathrm{B} 7$ & + & $1 / 1$ & $\mathrm{~S}$ \\
Ensino & 1 & $\mathrm{~B} 7 \rightarrow \mathrm{B} 8 \rightarrow \mathrm{B} 9$ & $+0+++$ & $4 / 5$ & $\mathrm{~S}$ \\
Sonda & 1 & $\mathrm{~B} 7 \rightarrow \mathrm{B} 8 \rightarrow \mathrm{B} 9$ & + & $1 / 1$ & $\mathrm{~S}$ \\
\hline
\end{tabular}

Nota. $\mathrm{O}$ sinal + corresponde ao acerto e o sinal 0 corresponde a outro tipo de responder sequencial.

Tabela 4 - Primeiro ensino e sonda de cada trio de PRI conjunto A.

\begin{tabular}{cccccc}
\hline $\begin{array}{c}\text { Tipo de bloco na } \\
\text { ordem de } \\
\text { apresentação }\end{array}$ & $\begin{array}{c}\text { Número de } \\
\text { apresentação do } \\
\text { bloco na sessão }\end{array}$ & $\begin{array}{c}\text { Tipo de tentativa } \\
\text { na ordem de } \\
\text { apresentação }\end{array}$ & $\begin{array}{c}\text { Resposta por } \\
\text { tentativa na } \\
\text { ordem de } \\
\text { apresentação }\end{array}$ & $\begin{array}{c}\text { Número de } \\
\text { acertos } \\
\text { sobre } 0 \\
\text { número de } \\
\text { tentativas }\end{array}$ & $\begin{array}{c}\text { Atingiu o } \\
\text { critério de } \\
\text { acerto }\end{array}$ \\
\hline Ensino & 1 & $\mathrm{~A} 1 \rightarrow \mathrm{A} 2 \rightarrow \mathrm{A} 3$ & $0+++$ & $3 / 4$ & $\mathrm{~S}$ \\
Sonda & 1 & $\mathrm{~A} 1 \rightarrow \mathrm{A} 2 \rightarrow \mathrm{A} 3$ & + & $1 / 1$ & $\mathrm{~S}$ \\
Ensino & 1 & $\mathrm{~A} 3 \rightarrow \mathrm{A} 4 \rightarrow \mathrm{A} 5$ & $000+++$ & $3 / 6$ & $\mathrm{~S}$ \\
Sonda & 1 & $\mathrm{~A} 3 \rightarrow \mathrm{A} 4 \rightarrow \mathrm{A} 5$ & + & $1 / 1$ & $\mathrm{~S}$ \\
Ensino & 1 & $\mathrm{~A} 5 \rightarrow \mathrm{A} 6 \rightarrow \mathrm{A} 7$ & $0+++$ & $3 / 4$ & $\mathrm{~S}$ \\
Sonda & 1 & $\mathrm{~A} 5 \rightarrow \mathrm{A} 6 \rightarrow \mathrm{A} 7$ & + & $1 / 1$ & $\mathrm{~S}$ \\
Ensino & 1 & $\mathrm{~A} 7 \rightarrow \mathrm{A} 8 \rightarrow \mathrm{A} 9$ & $0+++$ & $3 / 4$ & $\mathrm{~S}$ \\
Sonda & 1 & $\mathrm{~A} 7 \rightarrow \mathrm{A} 8 \rightarrow \mathrm{A} 9$ & + & $1 / 1$ & $\mathrm{~S}$ \\
\hline
\end{tabular}

Nota. $\mathrm{O}$ sinal + corresponde ao acerto e o sinal 0 corresponde a outro tipo de responder sequencial. 
Tabela 5 - Primeiro ensino e sonda de cada trio de PRI do Conjunto B.

\begin{tabular}{cccccc}
\hline $\begin{array}{c}\text { Tipo de bloco na } \\
\text { ordem de } \\
\text { apresentação }\end{array}$ & $\begin{array}{c}\text { Número de } \\
\text { apresentação do } \\
\text { bloco na sessão }\end{array}$ & $\begin{array}{c}\text { Tipo de tentativa } \\
\text { na ordem de } \\
\text { apresentação }\end{array}$ & $\begin{array}{c}\text { Resposta por } \\
\text { tentativa na } \\
\text { ordem de } \\
\text { apresentação }\end{array}$ & $\begin{array}{c}\text { Número de } \\
\text { acertos } \\
\text { sobre } 0 \\
\text { número de } \\
\text { tentativas }\end{array}$ & $\begin{array}{c}\text { Atingiu o } \\
\text { critério de } \\
\text { acerto }\end{array}$ \\
\hline Ensino & 1 & $\mathrm{~B} 1 \rightarrow \mathrm{B} 2 \rightarrow \mathrm{B} 3$ & +++ & $3 / 3$ & $\mathrm{~S}$ \\
Sonda & 1 & $\mathrm{~B} 1 \rightarrow \mathrm{B} 2 \rightarrow \mathrm{B} 3$ & + & $1 / 1$ & $\mathrm{~S}$ \\
Ensino & 1 & $\mathrm{~B} 3 \rightarrow \mathrm{B} 4 \rightarrow \mathrm{B} 5$ & +++ & $3 / 3$ & $\mathrm{~S}$ \\
Sonda & 1 & $\mathrm{~B} 3 \rightarrow \mathrm{B} 4 \rightarrow \mathrm{B} 5$ & + & $1 / 1$ & $\mathrm{~S}$ \\
Ensino & 1 & $\mathrm{~B} 5 \rightarrow \mathrm{B} 6 \rightarrow \mathrm{B} 7$ & $++0+++$ & $5 / 6$ & $\mathrm{~S}$ \\
Sonda & 1 & $\mathrm{~B} 5 \rightarrow \mathrm{B} 6 \rightarrow \mathrm{B} 7$ & + & $1 / 1$ & $\mathrm{~S}$ \\
Ensino & 1 & $\mathrm{~B} 7 \rightarrow \mathrm{B} 8 \rightarrow \mathrm{B} 9$ & +++ & $3 / 3$ & $\mathrm{~S}$ \\
Sonda & 1 & $\mathrm{~B} 7 \rightarrow \mathrm{B} 8 \rightarrow \mathrm{B} 9$ & + & $1 / 1$ & $\mathrm{~S}$ \\
\hline
\end{tabular}

Nota. $\mathrm{O}$ sinal + corresponde ao acerto e o sinal 0 corresponde a outro tipo de responder sequencial.

Tabela 6 - Primeiro ensino e sonda de cada trio de VAN do Conjunto A.

\begin{tabular}{cccccc}
\hline $\begin{array}{c}\text { Tipo de bloco na } \\
\text { ordem de } \\
\text { apresentação }\end{array}$ & $\begin{array}{c}\text { Número de } \\
\text { apresentação do } \\
\text { bloco na sessão }\end{array}$ & $\begin{array}{c}\text { Tipo de tentativa } \\
\text { na ordem de } \\
\text { apresentação }\end{array}$ & $\begin{array}{c}\text { Resposta por } \\
\text { tentativa na } \\
\text { ordem de } \\
\text { apresentação }\end{array}$ & $\begin{array}{c}\text { Número de } \\
\text { acertos } \\
\text { sobre } 0 \\
\text { número de } \\
\text { tentativas }\end{array}$ & $\begin{array}{c}\text { Atingiu o } \\
\text { critério de } \\
\text { acerto }\end{array}$ \\
\hline Ensino & 1 & $\mathrm{~A} 1 \rightarrow \mathrm{A} 2 \rightarrow \mathrm{A} 3$ & $00+++$ & $3 / 5$ & $\mathrm{~S}$ \\
Sonda & 1 & $\mathrm{~A} 1 \rightarrow \mathrm{A} 2 \rightarrow \mathrm{A} 3$ & + & $1 / 1$ & $\mathrm{~S}$ \\
Ensino & 1 & $\mathrm{~A} 3 \rightarrow \mathrm{A} 4 \rightarrow \mathrm{A} 5$ & $0+++$ & $3 / 4$ & $\mathrm{~S}$ \\
Sonda & 1 & $\mathrm{~A} 3 \rightarrow \mathrm{A} 4 \rightarrow \mathrm{A} 5$ & + & $1 / 1$ & $\mathrm{~S}$ \\
Ensino & 1 & $\mathrm{~A} 5 \rightarrow \mathrm{A} 6 \rightarrow \mathrm{A} 7$ & $0+++$ & $3 / 4$ & $\mathrm{~S}$ \\
Sonda & 1 & $\mathrm{~A} 5 \rightarrow \mathrm{A} 6 \rightarrow \mathrm{A} 7$ & + & $1 / 1$ & $\mathrm{~S}$ \\
Ensino & 1 & $\mathrm{~A} 7 \rightarrow \mathrm{A} 8 \rightarrow \mathrm{A} 9$ & +++ & $3 / 3$ & $\mathrm{~S}$ \\
Sonda & 1 & $\mathrm{~A} 7 \rightarrow \mathrm{A} 8 \rightarrow \mathrm{A} 9$ & + & $1 / 1$ & $\mathrm{~S}$ \\
\hline
\end{tabular}

Nota. O sinal + corresponde ao acerto e o sinal 0 corresponde a outro tipo de responder sequencial.

Tabela 7 - Primeiro ensino e sonda de cada trio de VAN do Conjunto B.

\begin{tabular}{cccccc}
\hline $\begin{array}{c}\text { Tipo de bloco na } \\
\text { ordem de } \\
\text { apresentação }\end{array}$ & $\begin{array}{c}\text { Número de } \\
\text { apresentação do } \\
\text { bloco na sessão }\end{array}$ & $\begin{array}{c}\text { Tipo de tentativa na } \\
\text { ordem de } \\
\text { apresentação }\end{array}$ & $\begin{array}{c}\text { Resposta por } \\
\text { tentativa na ordem } \\
\text { de apresentação }\end{array}$ & $\begin{array}{c}\text { Número de } \\
\text { acertos sobre } \\
\text { o número de } \\
\text { tentativas }\end{array}$ & $\begin{array}{c}\text { Atingiu o } \\
\text { critério de } \\
\text { acerto }\end{array}$ \\
\hline Ensino & 1 & $\mathrm{~B} 1 \rightarrow \mathrm{B} 2 \rightarrow \mathrm{B} 3$ & +++ & $3 / 3$ & $\mathrm{~S}$ \\
Sonda & 1 & $\mathrm{~B} 1 \rightarrow \mathrm{B} 2 \rightarrow \mathrm{B} 3$ & + & $1 / 1$ & $\mathrm{~S}$ \\
Ensino & 1 & $\mathrm{~B} 3 \rightarrow \mathrm{B} 4 \rightarrow \mathrm{B} 5$ & $0+++$ & $3 / 4$ & $\mathrm{~S}$ \\
Sonda & 1 & $\mathrm{~B} 3 \rightarrow \mathrm{B} 4 \rightarrow \mathrm{B} 5$ & + & $1 / 1$ & $\mathrm{~S}$ \\
Ensino & 1 & $\mathrm{~B} 5 \rightarrow \mathrm{B} 6 \rightarrow \mathrm{B} 7$ & $++0+++$ & $5 / 6$ & $\mathrm{~S}$ \\
Sonda & 1 & $\mathrm{~B} 5 \rightarrow \mathrm{B} 6 \rightarrow \mathrm{B} 7$ & + & $1 / 1$ & $\mathrm{~S}$ \\
Ensino & 1 & $\mathrm{~B} 7 \rightarrow \mathrm{B} 8 \rightarrow \mathrm{B} 9$ & +++ & $3 / 3$ & $\mathrm{~S}$ \\
Sonda & 1 & $\mathrm{~B} 7 \rightarrow \mathrm{B} 8 \rightarrow \mathrm{B} 9$ & + & $1 / 1$ & $\mathrm{~S}$ \\
\hline
\end{tabular}

Nota. O sinal + corresponde ao acerto e o sinal 0 corresponde a outro tipo de responder sequencial. 
No ensino dos blocos intermediários, cujo critério era de duas corretas consecutivas, o participante LEX precisou de 1 tentativa (1/1), no total de um bloco, para alcançar o critério no primeiro bloco intermediário $(\mathrm{A} 1 \rightarrow \mathrm{A} 2 \rightarrow \mathrm{A} 3$, $\mathrm{A} 3 \rightarrow \mathrm{A} 4 \rightarrow \mathrm{A} 5)$, e de 2 tentativas (1/2), no total de dois blocos, para o segundo bloco intermediário $(\mathrm{A} 1 \rightarrow \mathrm{A} 2 \rightarrow \mathrm{A} 3, \quad \mathrm{~A} 3 \rightarrow \mathrm{A} 4 \rightarrow \mathrm{A} 5$, $\mathrm{A} 5 \rightarrow \mathrm{A} 6 \rightarrow \mathrm{A} 7)$ e 1 tentativa (1/1), no total de um bloco para atingir o critério no terceiro bloco intermediário $(\rightarrow \mathrm{A} 2 \rightarrow \mathrm{A} 3, \mathrm{~A} 3 \rightarrow \mathrm{A} 4 \rightarrow \mathrm{A} 5$, $\mathrm{A} 5 \rightarrow \mathrm{A} 6 \rightarrow \mathrm{A} 7, \mathrm{~A} 7 \rightarrow \mathrm{A} 8 \rightarrow \mathrm{A} 9)$. Os participantes PRI e VAN precisaram de 1 tentativa (1/1), um único bloco, para alcançar o critério de cada bloco intermediário.

Nos blocos intermediários do Conjunto B todos os participantes precisaram de 1 tentativa (1/1), um único bloco, para alcançar o critério de cada bloco intermediário (ver Tabela 8 e 9).

\section{Sondas}

Os resultados obtidos através das tentativas de sonda dos oito trios apresentados (quatro de cada conjunto) para todos os participantes (LEX, PRI e VAN) demonstraram uma porcentagem de $100 \%$ de acerto imediato, alcançando o critério na primeira sonda de cada um dos trios (vide Tabelas 2, 3, 4, 5, 6 e 7).

Nas sondas feitas nos blocos intermediários, o participante LEX precisou de 1 tentativa (1/1), no total de um bloco, para alcançar o critério no primeiro bloco intermediário $(\mathrm{A} 1 \rightarrow \mathrm{A} 2 \rightarrow \mathrm{A} 3, \mathrm{~A} 3 \rightarrow \mathrm{A} 4 \rightarrow \mathrm{A} 5)$, e de 2 tentativas (1/2), no total de dois blocos, para o segundo bloco intermediário $(\mathrm{A} 1 \rightarrow \mathrm{A} 2 \rightarrow \mathrm{A} 3, \mathrm{~A} 3 \rightarrow \mathrm{A} 4 \rightarrow \mathrm{A} 5, \mathrm{~A} 5 \rightarrow \mathrm{A} 6 \rightarrow \mathrm{A} 7)$ e 1 tentativa (1/1), no total de um bloco para atingir o critério no terceiro bloco intermediário $(\rightarrow \mathrm{A} 2 \rightarrow \mathrm{A} 3, \quad \mathrm{~A} 3 \rightarrow \mathrm{A} 4 \rightarrow \mathrm{A} 5, \quad \mathrm{~A} 5 \rightarrow \mathrm{A} 6 \rightarrow \mathrm{A} 7$, A7 $\rightarrow$ A8 $\rightarrow$ A9). Os participantes PRI e VAN precisaram de 1 tentativa (1/1), um único bloco, para alcançar o critério de cada bloco intermediário (ver Tabela 10).

Nas sondas nos blocos intermediários do Conjunto $\mathrm{B}$, todos os participantes precisaram de 1 tentativa (1/1), um único bloco, para alcançar o critério de cada bloco intermediário (ver Tabela 11).

Tabela 8 - Número de tentativas necessárias para alcançar o critério no ensino dos blocos intermediários do Conjunto A dos participantes LEX, PRI e VAN.

\begin{tabular}{cccc}
\hline $\begin{array}{c}\text { Ensino Blocos } \\
\text { Intermediários }\end{array}$ & LEX & $\mathrm{N}^{\circ}$ de tentativas por participante & VAN \\
\hline $1^{\circ}$ Bloco & 1 & PRI & 1 \\
$2^{\circ}$ Bloco & 2 & 1 & 1 \\
$3^{\circ}$ Bloco & 1 & 1 & 1 \\
\hline $1^{\circ} \mathrm{Bl}$ & 1 &
\end{tabular}

Nota. $1^{\circ}$ Bloco $=\mathrm{A} 1 \rightarrow \mathrm{A} 2 \rightarrow \mathrm{A} 3, \mathrm{~A} 3 \rightarrow \mathrm{A} 4 \rightarrow \mathrm{A} 5.2^{\circ}$ Bloco $=\mathrm{A} 1 \rightarrow \mathrm{A} 2 \rightarrow \mathrm{A} 3, \mathrm{~A} 3 \rightarrow \mathrm{A} 4 \rightarrow \mathrm{A} 5, \mathrm{~A} 5 \rightarrow \mathrm{A} 6 \rightarrow \mathrm{A} 7.3^{\circ}$ $\mathrm{Bloco}=\mathrm{A} 1 \rightarrow \mathrm{A} 2 \rightarrow \mathrm{A} 3, \mathrm{~A} 3 \rightarrow \mathrm{A} 4 \rightarrow \mathrm{A} 5, \mathrm{~A} 5 \rightarrow \mathrm{A} 6 \rightarrow \mathrm{A} 7, \mathrm{~A} 7 \rightarrow \mathrm{A} 8 \rightarrow \mathrm{A} 9$.

Tabela 9 - Número de tentativas necessárias para alcançar o critério no ensino dos blocos intermediários do Conjunto B dos participantes LEX, PRI e VAN.

\begin{tabular}{cccc}
\hline $\begin{array}{c}\text { Ensino Blocos } \\
\text { Intermediários }\end{array}$ & LEX & $\begin{array}{c}\mathrm{N}^{0} \text { de tentativas por participante } \\
\text { PRI }\end{array}$ & VAN \\
\hline $1^{0}$ Bloco & 1 & 1 & 1 \\
$2^{0}$ Bloco & 1 & 1 & 1 \\
$3^{\circ}$ Bloco & 1 & 1 & 1 \\
\hline
\end{tabular}

Nota. $1^{\circ}$ Bloco $=\mathrm{B} 1 \rightarrow \mathrm{B} 2 \rightarrow \mathrm{B} 3, \mathrm{~B} 3 \rightarrow \mathrm{B} 4 \rightarrow \mathrm{B} 5.2^{\circ}$ Bloco $=\mathrm{B} 1 \rightarrow \mathrm{B} 2 \rightarrow \mathrm{B} 3, \mathrm{~B} 3 \rightarrow \mathrm{B} 4 \rightarrow \mathrm{B} 5, \mathrm{~B} 5 \rightarrow \mathrm{B} 6 \rightarrow \mathrm{B} 7.3^{\circ}$ B loco $=\mathrm{B} 1 \rightarrow \mathrm{B} 2 \rightarrow \mathrm{B} 3, \mathrm{~B} 3 \rightarrow \mathrm{B} 4 \rightarrow \mathrm{B} 5, \mathrm{~B} 5 \rightarrow \mathrm{A} 6 \rightarrow \mathrm{B} 7, \mathrm{~B} 7 \rightarrow \mathrm{B} 8 \rightarrow \mathrm{B} 9$. 
Tabela 10 - Número de tentativas necessárias para alcançar o critério nas sondas dos blocos intermediários do conjunto A dos participantes LEX, PRI e VAN.

\begin{tabular}{cccc}
\hline Sondas Blocos Intermediários & \multicolumn{3}{c}{$\mathrm{N}^{0}$ de tentativas por participante } \\
& LEX & PRI & VAN \\
\hline $1^{\circ}$ Bloco & 1 & 1 & 1 \\
$2^{\circ}$ Bloco & 2 & 1 & 1 \\
$3^{\circ}$ Bloco & 1 & 1 & 1 \\
\hline
\end{tabular}

Nota. $1^{\circ}$ Bloco $=\mathrm{A} 1 \rightarrow \mathrm{A} 2 \rightarrow \mathrm{A} 3, \mathrm{~A} 3 \rightarrow \mathrm{A} 4 \rightarrow \mathrm{A} 5.2^{\circ} \mathrm{Bloco}=\mathrm{A} 1 \rightarrow \mathrm{A} 2 \rightarrow \mathrm{A} 3, \mathrm{~A} 3 \rightarrow \mathrm{A} 4 \rightarrow \mathrm{A} 5, \mathrm{~A} 5 \rightarrow \mathrm{A} 6 \rightarrow \mathrm{A} 7.3^{\circ}$ $\mathrm{Bloco}=\mathrm{A} 1 \rightarrow \mathrm{A} 2 \rightarrow \mathrm{A} 3, \mathrm{~A} 3 \rightarrow \mathrm{A} 4 \rightarrow \mathrm{A} 5, \mathrm{~A} 5 \rightarrow \mathrm{A} 6 \rightarrow \mathrm{A} 7, \mathrm{~A} 7 \rightarrow \mathrm{A} 8 \rightarrow \mathrm{A} 9$.

Tabela 11 - Número de tentativas necessárias para alcançar o critério nas sondas dos blocos intermediários do Conjunto B dos participantes LEX, PRI e VAN.

\begin{tabular}{cccc}
\hline $\begin{array}{c}\text { Ensino Blocos } \\
\text { Intermediários }\end{array}$ & LEX & $\mathrm{N}^{0}$ de tentativas por participante & \\
\hline $1^{\circ}$ Bloco & 1 & PRI & VAN \\
$2^{\circ}$ Bloco & 1 & 1 & 1 \\
$3^{\circ}$ Bloco & 1 & 1 & 1 \\
\hline ta $1^{\circ}$ Bloco $=\mathrm{B} 1 \rightarrow \mathrm{B} 2 \rightarrow \mathrm{B} 3, \mathrm{~B} 3 \rightarrow \mathrm{B} 4 \rightarrow \mathrm{B} 5.2^{\circ} \mathrm{Bloco}=\mathrm{B} 1 \rightarrow \mathrm{B} 2 \rightarrow \mathrm{B} 3, \mathrm{~B} 3 \rightarrow \mathrm{B} 4 \rightarrow \mathrm{B} 5, \mathrm{~B} 5 \rightarrow \mathrm{B} 6 \rightarrow \mathrm{B} 7.3^{\circ} \mathrm{B}$ loco \\
$\mathrm{B} 1 \rightarrow \mathrm{B} 2 \rightarrow \mathrm{B} 3, \mathrm{~B} 3 \rightarrow \mathrm{B} 4 \rightarrow \mathrm{B} 5, \mathrm{~B} 5 \rightarrow \mathrm{B} 6 \rightarrow \mathrm{B} 7, \mathrm{~B} 7 \rightarrow \mathrm{B} 8 \rightarrow \mathrm{B} 9$.
\end{tabular}

\section{Teste de transitividade}

Os três participantes apresentaram acertos nos testes de transitividade. No primeiro teste de transitividade com o conjunto A, todos os participantes acertaram $100 \%$ nas três sequências programadas. Porém, no segundo teste, com o conjunto $\mathrm{B}$, o participante LEX acertou duas sequências de um total de três sequências, equivocando-se no trio B5 $\rightarrow$ B7 $\rightarrow$ B9. Já os participantes PRI e VAN responderam com $100 \%$ de acerto nas três sequências programadas desse conjunto de estímulos, podendo ser observada a emergência de relações transitivas com os três elementos presentes (vide Tabelas 12 e 13).

\section{Teste de conectividade (substituibilidade dos estímulos) dos Conjuntos A/B}

Nos testes de conectividade, todos os participantes responderam corretamente a quatro sequências similares (que iniciavam com numerais ou formas não representacionais) dentre as seis sequências programadas, evidenciando uma emergência parcial de classes ordinais mais longas.

Tabela 12 - Desempenho dos participantes nos testes de transitividade do conjunto A com tipo de tentativa e número de respostas corretas pelo número de tentativas.

\begin{tabular}{ccc}
\hline \multirow{2}{*}{ Participantes } & $\begin{array}{c}\text { Tipo de tentativa } \\
\text { na ordem de apresentação }\end{array}$ & $\begin{array}{c}\text { Número de respostas } \\
\text { corretas pelo número de } \\
\text { tentativas }\end{array}$ \\
\hline \multirow{2}{*}{ LEX } & $\mathrm{A} 1 \rightarrow \mathrm{A} 3 \rightarrow \mathrm{A} 5$ & $1 / 1$ \\
& $\mathrm{~A} 3 \rightarrow \mathrm{A} 5 \rightarrow \mathrm{A} 7$ & $1 / 1$ \\
$\mathrm{~A} 5 \rightarrow \mathrm{A} 7 \rightarrow \mathrm{A} 9$ & $1 / 1$ \\
$\mathrm{~A} 1 \rightarrow \mathrm{A} 3 \rightarrow \mathrm{A} 5$ & $1 / 1$ \\
$\mathrm{ARI}$ & $\mathrm{A} 3 \rightarrow \mathrm{A} 5 \rightarrow \mathrm{A} 7$ & $1 / 1$ \\
& $\mathrm{~A} 5 \rightarrow \mathrm{A} 7 \rightarrow \mathrm{A} 9$ & $1 / 1$ \\
$\mathrm{~A} 1 \rightarrow \mathrm{A} 3 \rightarrow \mathrm{A} 5$ & $1 / 1$ \\
& $\mathrm{~A} 3 \rightarrow \mathrm{A} 5 \rightarrow \mathrm{A} 7$ & $1 / 1$ \\
& $\mathrm{~A} 5 \rightarrow \mathrm{A} 7 \rightarrow \mathrm{A} 9$ & $1 / 1$ \\
\hline
\end{tabular}


Tabela 13 - Desempenho dos participantes nos testes de transitividade do conjunto B com tipo de tentativa e número de respostas corretas pelo número de tentativas.

\begin{tabular}{ccc}
\hline Participantes & $\begin{array}{c}\text { Tipo de tentativa } \\
\text { na ordem de apresentação }\end{array}$ & $\begin{array}{c}\text { Número de respostas } \\
\text { corretas pelo número de } \\
\text { tentativas }\end{array}$ \\
\hline \multirow{3}{*}{ LEX } & $\mathrm{B} 1 \rightarrow \mathrm{B} 3 \rightarrow \mathrm{B} 5$ & $1 / 1$ \\
& $\mathrm{~B} 3 \rightarrow \mathrm{B} 5 \rightarrow \mathrm{B} 7$ & $1 / 1$ \\
$\mathrm{~B} 5 \rightarrow \mathrm{B} 7 \rightarrow \mathrm{B} 9$ & $0 / 1$ \\
$\mathrm{BRI}$ & $\mathrm{B} 1 \rightarrow \mathrm{B} 3 \rightarrow \mathrm{B} 5$ & $1 / 1$ \\
& $\mathrm{~B} 3 \rightarrow \mathrm{B} 5 \rightarrow \mathrm{B} 7$ & $1 / 1$ \\
& $\mathrm{~B} 5 \rightarrow \mathrm{B} 7 \rightarrow \mathrm{B} 9$ & $1 / 1$ \\
VAN & $\mathrm{B} 1 \rightarrow \mathrm{B} 3 \rightarrow \mathrm{B} 5$ & $1 / 1$ \\
& $\mathrm{~B} 3 \rightarrow \mathrm{B} 5 \rightarrow \mathrm{B} 7$ & $1 / 1$ \\
& $\mathrm{~B} 5 \rightarrow \mathrm{B} 7 \rightarrow \mathrm{B} 9$ & $1 / 1$ \\
\hline
\end{tabular}

Tabela 14 - Desempenho dos participantes nos testes de conectividade com tipo de tentativa e número de respostas corretas pelo número de tentativas.

\begin{tabular}{ccc}
\hline Participantes & $\begin{array}{c}\text { Tipo de tentativa } \\
\text { na ordem de apresentação }\end{array}$ & $\begin{array}{c}\text { Número de respostas } \\
\text { corretas pelo número de } \\
\text { tentativas }\end{array}$ \\
\hline \multirow{3}{*}{ LEX } & $\mathrm{A} 1 \rightarrow \mathrm{B} 2 \rightarrow \mathrm{A} 3$ & $1 / 1$ \\
$\mathrm{~B} 4 \rightarrow \mathrm{A} 5 \rightarrow \mathrm{B} 6$ & $0 / 1$ \\
$\mathrm{~A} 7 \rightarrow \mathrm{B} 8 \rightarrow \mathrm{A} 9$ & $1 / 1$ \\
$\mathrm{~B} 1 \rightarrow \mathrm{A} 2 \rightarrow \mathrm{B} 3$ & \\
$\mathrm{~A} 4 \rightarrow \mathrm{B} 5 \rightarrow \mathrm{A} 6$ & $1 / 1$ \\
$\mathrm{~B} 7 \rightarrow \mathrm{A} 8 \rightarrow \mathrm{B} 9$ & $0 / 1$ \\
$\mathrm{~A} 1 \rightarrow \mathrm{B} 2 \rightarrow \mathrm{A} 3$ & $1 / 1$ \\
$\mathrm{~B} 4 \rightarrow \mathrm{A} 5 \rightarrow \mathrm{B} 6$ & $1 / 1$ \\
$\mathrm{~A} 7 \rightarrow \mathrm{B} 8 \rightarrow \mathrm{A} 9$ & $0 / 1$ \\
& $1 / 1$ \\
$\mathrm{~B} 1 \rightarrow \mathrm{A} 2 \rightarrow \mathrm{B} 3$ & \\
$\mathrm{~A} 4 \rightarrow \mathrm{B} 5 \rightarrow \mathrm{A} 6$ & $1 / 1$ \\
$\mathrm{~B} 7 \rightarrow \mathrm{A} 8 \rightarrow \mathrm{B} 9$ & $0 / 1$ \\
$\mathrm{~A} 1 \rightarrow \mathrm{B} 2 \rightarrow \mathrm{A} 3$ & $1 / 1$ \\
$\mathrm{~B} 4 \rightarrow \mathrm{A} 5 \rightarrow \mathrm{B} 6$ & $1 / 1$ \\
$\mathrm{~A} 7 \rightarrow \mathrm{B} 8 \rightarrow \mathrm{A} 9$ & $0 / 1$ \\
& $\mathrm{~B} 1 \rightarrow \mathrm{A} 2 \rightarrow \mathrm{B} 3$ & $1 / 1$ \\
$\mathrm{~A} 4 \rightarrow \mathrm{B} 5 \rightarrow \mathrm{A} 6$ & $1 / 1$ \\
$\mathrm{~B} 7 \rightarrow \mathrm{A} 8 \rightarrow \mathrm{B} 9$ & $0 / 1$ \\
& & $1 / 1$ \\
\hline
\end{tabular}

\section{Teste de Generalização}

Todos os participantes ordenaram os tapetes de EVA contendo numerais de um a nove encaixando-os corretamente da esquerda para a direita. Além disso, ordenaram corretamente todas as peças nas sequências lógicas previstas no jogo de encaixe com as peças em madeira usadas na fase de pré-testes.

\section{Discussão}

Os resultados demonstraram o efeito do procedimento de ensino por sobreposição de trios de estímulos numéricos sobre a emergência de relações ordinais em préescolares. Entretanto, respostas não previstas experimentalmente para a primeira tentativa dos trios apresentados podem sugerir, pelo menos, duas questões passíveis de discussão. 
A primeira diz respeito à reversão da função de estímulos em um mesmo conjunto (ou entre conjuntos diferentes nos casos dos testes de conectividade), na qual o estímulo comum aos trios sobrepostos exerceu a função de terceiro e, em seguida, de primeiro (ex. $\mathrm{B} 3 \rightarrow \mathrm{B} 4 \rightarrow \mathrm{B} 5)$. A segunda questão refere-se a um possível controle exercido pela novidade dos estímulos apresentados. Nesse caso, após aprender a sequência de três estímulos, por exemplo, $\mathrm{A} 1 \rightarrow \mathrm{A} 2 \rightarrow \mathrm{A} 3$, é apresentado ao participante o trio subsequente A3 $\rightarrow$ A $4 \rightarrow$ A5 . Assim, o estímulo já conhecido, o A3, pode ser excluído em detrimento dos dois estímulos novos restantes que podem ser selecionados primeiramente.

Uma possível explicação para resultados mais consistentes no conjunto "B", provavelmente, deve-se à manutenção dessa variável (uma única forma não representacional) diferentemente da quantidade de numerais de 1 a 9 usados no conjunto "A" variando a disposição espacial e o tamanho da mesma forma não representacional.

Os testes de transitividade mostraram resultados bastante acima da linha do acaso. Todos os participantes apresentaram porcentagens acima de $80 \%$ de acerto. Tais testes de transitividade mostraram a utilidade do procedimento em produzir novas sequências intraconjunto sem treino direto (cf. Holcomb et al., 1997). Por isso, Stromer e Mackay (1993) afirmaram que as relações derivadas das contingências de ensino por sobreposição de estímulos podem ser consideradas relações verdadeiramente transitivas.

Assim como Souza et al. (2005), no presente estudo foram testadas relações entre estímulos que nunca foram apresentados juntos na fase de treino, configurando a emergência de relações de ordem e a formação de classes ordinais entre estímulos de sequências ensinadas separadamente (Stromer \& Mackay, 1993).

A configuração dos testes de substituição múltipla usados no procedimento, nos quais mais de um estímulo de um mesmo conjunto é apresentado junto a outro de conjunto diferente, pode amparar a hipótese explicativa para as respostas incorretas dos participantes às sequências apresentadas no teste de conectividade. $\mathrm{O}$ controle pelos seus conjuntos correspondentes pode ter ocorrido assim como em Stromer e Mackay (1993).
Se, por um lado, o ensino por sobreposição de três estímulos soluciona o problema da escolha de estímulos por exclusão existente no ensino através de sobreposição de pares de estímulos, por outro lado, pode aumentar a margem de erro da criança, ao haver mais de uma possibilidade de ordenação, além de aumentar a complexidade da produção de sequências comportamentais pelo número de estímulos apresentados (de um a nove).

Os resultados obtidos nos pós-testes (testes de generalização) demonstraram a eficácia real desse novo arranjo de contingências de ensino por meio do procedimento de sobreposição de trios de estímulos sobre a emergência de relações ordinais, pois todos os participantes alcançaram acertos de $100 \%$ nesses testes.

\section{Referências}

Assis, G. J. A., Corrêa, D. R., Souza, C. B. A., \& Prado, P. S. T. (2010). Aprendizagem de relações ordinais por meio de treino de uma única sequência de estímulos. Psicologia: Teoria e Pesquisa, 26, 165-176.

Assis, G. J. A., Miccione, M. M., \& Nunes, A. L. M. (2010). Da produção de sequências comportamentais à equivalência de estímulos sequenciais. In J. S. Carmo \& P. S. T. Prado (Orgs.), Relações simbólicas e aprendizagem matemática (pp. 69-88). Santo André, SP: ESETEC.

Galy, E., Camps, J. F., \& Melan, C. (2003). Sequence class formation following learning of short sequences. The Psychological Record, 53, 635-645.

Green, G., Stromer, R., \& Mackay, H. (1993). Relational learning in stimulus sequences. The Psychological Record, 43, 599-616.

Holcomb, W. L., Stromer, R., \& Mackay, H. (1997). Transitivity and emergent sequence performance in young children. Journal of Experimental Child Psychology, 65, 96-124.

Lopes Júnior, J., \& Agostini, M. F. (2004). Aquisição e emergência de relações ordinais por crianças da segunda série do ensino fundamental. In O. M. P. R. Rodrigues, T. G. M. do Valle, A. C. M. Almeida-Verdu, J. Lopes Júnior \& M. R. Cavalcante (Eds.), Psicologia do desenvolvimento e aprendizagem: Investigações e análises (pp. 95-127). São Carlos: Rima. 
Maydak, M., Stromer, R., Mackay, H., \& Stoddard, L. (1995). Stimulus classes in matching to sample and sequence production: the emergence of numeric relations. Research in Developmental Disabilities, 16, 179-204.

Mcllvane, W. J., \& Dube, W. V. (2003). Stimulus control topography coherence theory: Foundations and extensions. The Behavior Analyst, 26, 195-213

Miccione, M. M., Assis, G. J. A., \& Costa, T. D. (2010). Variáveis de controle sobre o responder ordinal: Revisitando estudos empíricos. Revista Perspectivas em Análise do comportamento, 1(2), 130-148.

Nunes, A. L. M., \& Assis, G. J. A. (2006). Emergência de classes ordinais após o ensino de relações numéricas. Revista Brasileira de Análise do Comportamento, 2 , 203-219.

Ribeiro, M. P. L., Assis, G. J. A., \& Enumo, S. R. F. (2007). Comportamento matemático: Relações ordinais e inferência transitiva em pré-escolares. Psicologia: Teoria $e$ Pesquisa, 23, 25-32.

Santos, A. S L., Silva, A. M. M. V., Baptista, M. Q., \& Assis, G. J. A. (1997). REL 1.0: Sistema computadorizado para o ensino de discriminações simples e condicionais. Resumos de Comunicações Científicas (p.192), XXVII Reunião Anual de Psicologia, Sociedade Brasileira de Psicologia, Ribeirão Preto-SP.

Sidman, M. (1994). Equivalence relations and behavior: A research Story. Boston: Authors Cooperative Inc. Publishers.
Sigurdardottir, Z. G., Green, G., \& Saunders, R. R. (1990). Equivalence classes generated by sequence training. Journal of the Experimental Analysis of Behavior, 53, 4763.

Souza, R. D. C., Assis, G. J. A., \& Magalhães, P. G. S. (2005). Equivalência numérica em crianças surdas. Temas em Psicologia, 22, 113-127.

Souza, R. D. C., Assis, G. J. A., Magalhães, P. G. S., \& Prado, P. S. T. (2008). Efeitos de um procedimento de ensino de produção de sequências por sobreposição sob controle condicional em crianças surdas. Interação em Psicologia, 12, 59-75.

Stevens, J. (1951). Mathematics, measurement, and psychophysics. In S. Stevens (Org.), Handbook of experimental psychology (pp. 1-49). New York: John Wiley and Sons, Inc.

Stromer, R., \& Mackay, H. A. (1990). A note on the study of transitive relation in stimulus sequence. Experimental Analysis of Human Behavior Bulletin, 8, 2-5.

Stromer, R., \& Mackay, H. (1993). Human sequential behavior: relations among stimuli, class formation and derived sequences. The Psychological Record, 43, 107-131.

Verdu, A. C. M. A., Souza, D. G., \& Lopes Jr., J. (2006). Formação de classes ordinais após a aprendizagem de sequências independentes. Estudos de Psicologia, 11, 87-99.

Recebido em 14 de Novembro de 2011 Texto reformulado em 31 de Maio de 2012 Aceite em 29 de Junho de 2012 Publicado em 31 de Dezembro de 2012 